\title{
Strain states in semipolar III-nitride semiconductor quantum wells
}

\section{AUTHOR(S):}

Funato, M.; Inoue, D.; Ueda, M.; Kawakami, Y.; Narukawa, Y.; Mukai, T.

\section{CITATION:}

Funato, M....[et al]. Strain states in semipolar III-nitride semiconductor quantum wells. JOURNAL OF APPLIED PHYSICS 2010, 107(12): 123501.

\section{ISSUE DATE:}

2010-06

URL:

http://hdl.handle.net/2433/147194

\section{RIGHT:}

Copyright 2010 American Institute of Physics. This article may be downloaded for personal use only. Any other use requires prior permission of the author and the American Institute of Physics. The following article appeared in JOURNAL OF APPLIED PHYSICS107, 123501 (2010) and may be found at 


\title{
Strain states in semipolar III-nitride semiconductor quantum wells
}

\author{
M. Funato, ${ }^{1}$ D. Inoue, ${ }^{1}$ M. Ueda, ${ }^{1}$ Y. Kawakami, ${ }^{1}$ Y. Narukawa, ${ }^{2}$ and T. Mukai ${ }^{2}$ \\ ${ }^{1}$ Department of Electronic Science and Engineering, Kyoto University, Kyoto 615-8510, Japan \\ ${ }^{2}$ Nitride Semiconductor Research Laboratory, Nichia Corporation, Tokushima 774-8601, Japan
}

(Received 8 March 2010; accepted 7 May 2010; published online 16 June 2010)

\begin{abstract}
Strain states in wurtzite III-nitride semiconductor quantum wells (QWs) are investigated. X-ray diffraction (XRD) reciprocal space mapping using semipolar (11) 2 ) and (1 $\overline{1} 01)$ InGaN/GaN QWs as test samples demonstrates that the projections of reciprocal lattice vectors of unstrained GaN and pseudomorphically strained InGaN on the interface agrees, indicating continuity of layers across the interface. High resolution transmission electron microscopy supports the XRD analysis. Based on the experimental results, strain tensor elements are extracted for arbitrary crystalline orientation. Furthermore, expansion of the model to arbitrary crystal structures is suggested. () 2010 American Institute of Physics. [doi:10.1063/1.3446297]
\end{abstract}

\section{INTRODUCTION}

Strain in semiconductors plays a crucial role in determining the electronic band structures. ${ }^{1}$ For example, strained quantum wells (QWs) are widely used as light-emitting layers, where the fundamental properties such as transition energy, optical anisotropy, and optical gain are subject to strain. Therefore, to thoroughly understand the optical properties and to create novel functionalities, it is indispensable to identify the strain states in semiconductors. It is well known that the strain states in semiconductors are well described by the elasticity theory. ${ }^{2}$ However, a problem remains in boundary conditions at heterointerfaces; to date, the boundary conditions for well-defined, low index crystallographic planes, such as zincblende $\{001\},\{110\}$, and $\{111\}$, and wurtzite $\{0001\},\{1 \overline{1} 00\}$, and $\{11 \overline{2} 0\}$, have already been established, whereas those for planes with higher indices are still controversial, although such planes are receiving increased interest because of, for example, potential high emission quantum efficiencies from wurtzite, semipolar III-nitride semiconductors. ${ }^{3-12}$ The lack of experimentally or theoretically supported boundary conditions prevents us from unambiguously determining the strain states in heterostructures grown on high index surfaces.

There have been several proposals on the boundary conditions to describe strain not only for conventional zincblende III-V semiconductors ${ }^{13,14}$ but also for wurtzite III-nitride semiconductors. ${ }^{3-6}$ Refs. 3 and 14 are based on the same idea, which is the projections of the primitive translation vectors of the epilayer and substrate on the interface should coincide. On the other hand, Refs. 4-6 and 13 consider lattice mismatch within the growth plane, although several differences can be found in the assumptions and expressions; for example, Refs. 4, 5, and 13 continuously vary Euler angles, whereas Ref. 6 uses the more practical Miller indices. However, all of those reports are theoretical considerations, and decisive experimental evidence to clarify the strain states, particularly in planes with high Miller indices, has yet to be provided. Herein, we investigate strain in $(11 \overline{2} 2)$ and $(1 \overline{1} 01)$ InGaN/GaN QWs by x-ray diffraction (XRD) reciprocal space mapping (RSM) and transmission electron microscopy (TEM). The (11 $\overline{2} 2)$ plane is a semipolar plane promising for high emission efficiencies ${ }^{7,9}$ and lightemitting diodes fabricated on this plane demonstrated performances suitable for practical applications. ${ }^{10,11}$ Unfortunately, existing models could not reproduce the experimental results of XRD-RSM and TEM. Hence, we developed a new model for arbitrary crystallographic orientation of the wurtzite structure, and furthermore, suggested the application of the proposed model to arbitrary crystal structures.

\section{DEFINITIONS OF COORDINATES}

Here, two orthogonal coordinate systems are used. Figure 1 explains their definitions using the semipolar (11 2 2) plane as an example. The $(x, y, z)$ coordinates are defined with respect to the wurtzite lattice. The $x$ and $y$ axes are in the (0001) plane, while the $z$ axis is parallel to [0001]. The $x$ and $y$ axes can be aligned in two arbitrary perpendicular directions due to crystal symmetry, but are chosen to be parallel to $[\overline{1} \overline{1} 20]$ and $[1 \overline{1} 00]$, respectively, for definitiveness. On the other hand, the $\left(x^{\prime}, y^{\prime}, z^{\prime}\right)$ coordinates are defined with respect to the growth plane, where $x^{\prime}$ and $y^{\prime}$ lay in the plane, and $z^{\prime}$ is along the growth direction. For $(11 \overline{2} l)$ $[(1 \overline{1} 0 l)]$ planes, the $y^{\prime}\left[x^{\prime}\right]$ axis is defined to agree with the $y$ $[x]$ axis. Hence, rotation around the $y[x]$ axis yielded $\left(x^{\prime}, y^{\prime}, z^{\prime}\right)$ from $(x, y, z)$. The tilt of the $c$ axis in the semipolar plane from the surface normal, which corresponds to the

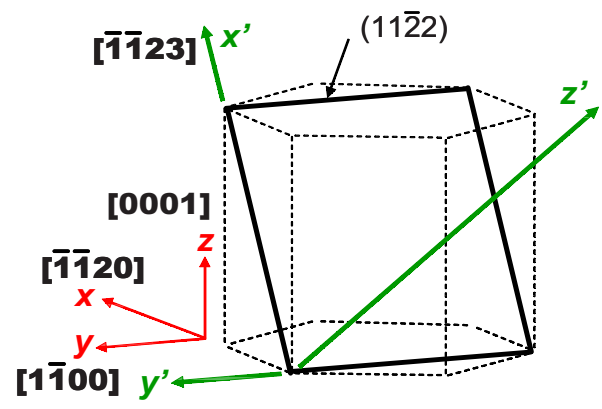

FIG. 1. (Color online) (11 22$)$ plane and definitions of the $(x, y, z)$ and $\left(x^{\prime}, y^{\prime}, z^{\prime}\right)$ coordinates. 

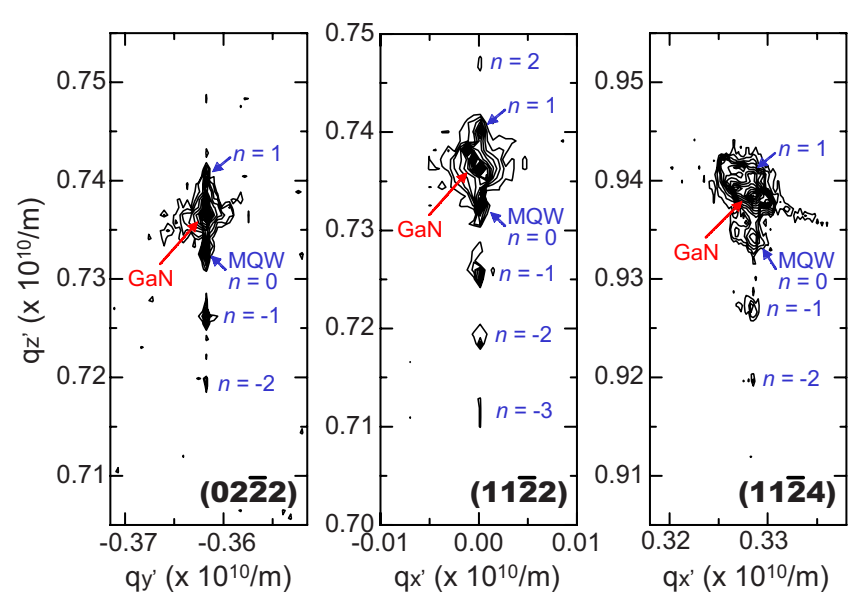

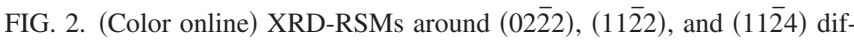
fractions of the (11/22) InGaN/GaN MQW.

angle between $z$ and $z^{\prime}$, is denoted by $\theta$. For (11 $\left.\overline{2} 2\right)$, $x^{\prime}\left\|[\overline{1} \overline{1} 23], y^{\prime}\right\|[1 \overline{1} 00], z^{\prime} \perp(11 \overline{2} 2)$, and $\theta=58^{\circ}$.

\section{EXPERIMENTAL RESULTS}

\section{A. XRD-RSM}

To assess the strain in semipolar InGaN/GaN QWs, five period multiple QWs (MQWs) with $3.4 \mathrm{~nm}$ thick InGaN wells were fabricated on $(11 \overline{2} 2) \mathrm{GaN}$ substrates by metalorganic vapor phase epitaxy. Details for growth are described elsewhere. ${ }^{9}$ Because the substrate was bulk GaN, only InGaN was compressively strained. XRD-RSMs were conducted for many crystallographic planes, and Fig. 2 shows typical results where the coordinate axes of $q_{x^{\prime}}, q_{y^{\prime}}$, and $q_{z^{\prime}}$ in the reciprocal space corresponds to the $x^{\prime}, y^{\prime}$, and $z^{\prime}$ axes in the real space, respectively. In all the RSMs, satellite diffraction spots labeled $n$ were clearly derived from the MQW periodicity.

In both the $q_{x^{\prime}}-q_{z^{\prime}}$ and $q_{y^{\prime}}-q_{z^{\prime}}$ planes, the diffraction spots from $\mathrm{GaN}$ and the MQW were vertically aligned along the $q_{z^{\prime}}$ axis, indicating that $(1 / d) \cos \varphi$ coincides between the MQW and GaN where $d$ and $\varphi$ designate the lattice spacing and the angle formed between the interface $\left(q_{x^{\prime}}-q_{y^{\prime}}\right.$ plane $)$ and reciprocal vector, respectively. Because $d / \cos \varphi$ is the layer spacing within the interface in real space, the vertically aligned diffraction spots guarantee continuity of the lattice planes across the interface. This relationship between the reciprocal space and the real space is schematically illustrated in Fig. 3(a). For the (11) 2 ) growth plane, $\varphi$ was $90^{\circ}$, and consequently, $d / \cos \varphi$ at the interface became infinity, indicating that the (11) 2 ) planes in InGaN and GaN are parallel, which is reasonable for interfaces with singular planes such as $(0001),\{11 \overline{2} 2\}$, and $\{1 \overline{1} 00\}$. From these conditions, a distorted InGaN lattice emerged, as drawn in Figs. 3(b) and 3(c) where the distortion is exaggerated to clearly show the difference between unstrained and strained lattices. The measured inclination between $\mathrm{InGaN}$ and $\mathrm{GaN}\left(=\left|\varphi_{1}-\varphi_{2}\right|\right)$ was on the order of $0.1^{\circ}$.
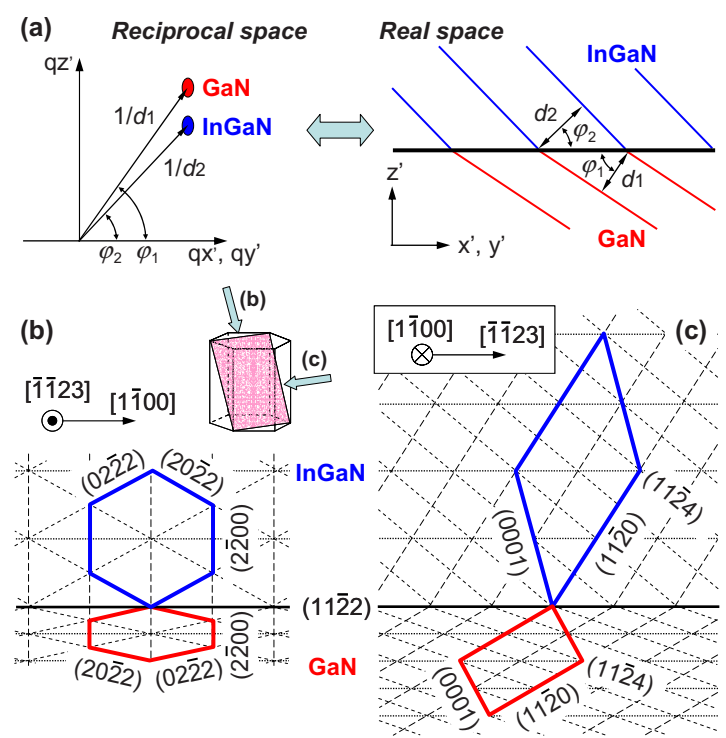

FIG. 3. (Color online) (a) Relationship between reciprocal and real spaces. Ellipsoidal regions in the reciprocal space express diffraction spots and lines in real space indicate corresponding lattice planes. (b) and (c) are schematics of strained $\mathrm{InGaN}$ grown pseudomorphically on unstrained $\mathrm{GaN}$ viewed along the [1 $\overline{1} 23]$ and [1 100$]$ directions, respectively. Inset between (b) and (c) shows the observation directions for the respective figures.

\section{B. TEM analysis}

To confirm the model proposed above, cross sectional TEM measurements were performed for a $2.5 \mathrm{~nm}$ thick InGaN single QW grown on a $\mathrm{GaN}(11 \overline{2} 2)$ substrate. The TEM specimens were prepared by focused ion beam sampling, and an acceleration voltage for TEM observations was $300 \mathrm{KV}$. Figures 4(a) and 4(b) display cross sectional high

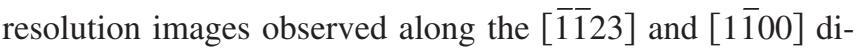
rections, respectively. In Fig. 4(b), spots like those in Fig. 4(a) were not observed because the TEM resolution was comparable to the $(11 \overline{2} 0)$ spacing $(0.159 \mathrm{~nm}$ for $\mathrm{GaN})$ and spots along the $[11 \overline{2} 0]$ direction could not be well resolved. Within these high resolution images, layer continuity between $\mathrm{GaN}$ and $\mathrm{InGaN}$ was confirmed. To visualize variations in layer spacing and inclination suggested by XRDRSM for the pseudomorphic interface, several representative lattice planes were analyzed through Fourier transform of the high resolution images. The high resolution images were first meshed with a $0.25 \mathrm{~nm}$ step, and then, at each gridding point, fast Fourier transform (FFT) was locally performed for a tiny region of $\sim 4 \times 4 \mathrm{~nm}^{2}$. This procedure produced local diffraction patterns, and the inset in Fig. 4(a) shows an example. The local diffraction pattern enabled the spacing and the normal direction of layers to be mapped. Hence, the mappings Figs. 4(c)-4(e) were derived from Fig. 4(a) by analyzing the respective (diffraction) spots designated in the inset FFT pattern, while the mapping Fig. 4(f) was obtained from Fig. 4(b). The rotation was defined as the angle of the normal vector in InGaN with respect to that in $\mathrm{GaN}$, and positive when rotation was counterclockwise.

FFT analyses clearly confirmed several important aspects of the lattice distortion model. For example, Fig. 3(b) predicted that the $(02 \overline{2} 2)$ plane in InGaN has a larger layer 

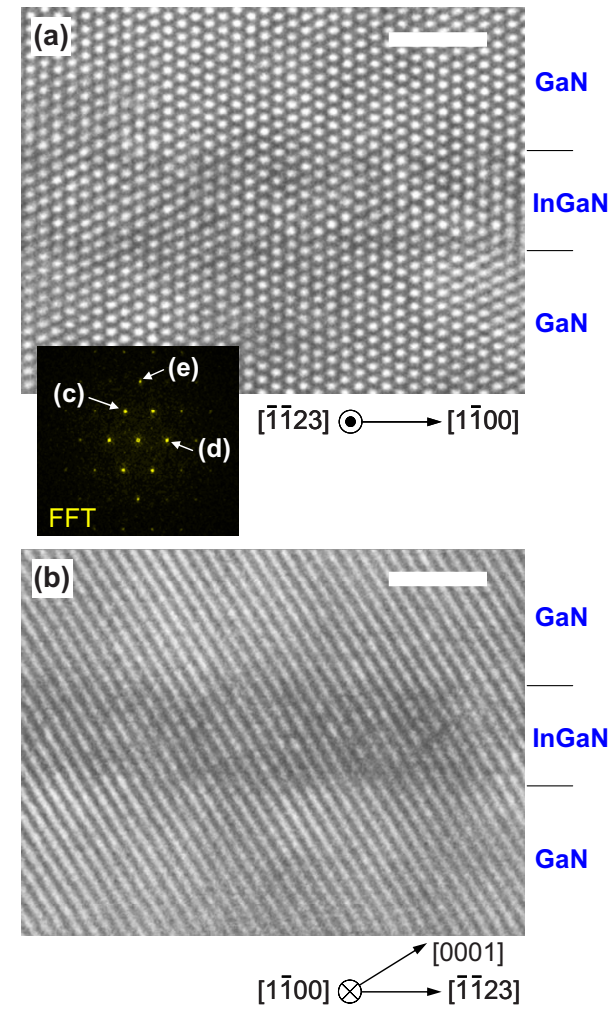
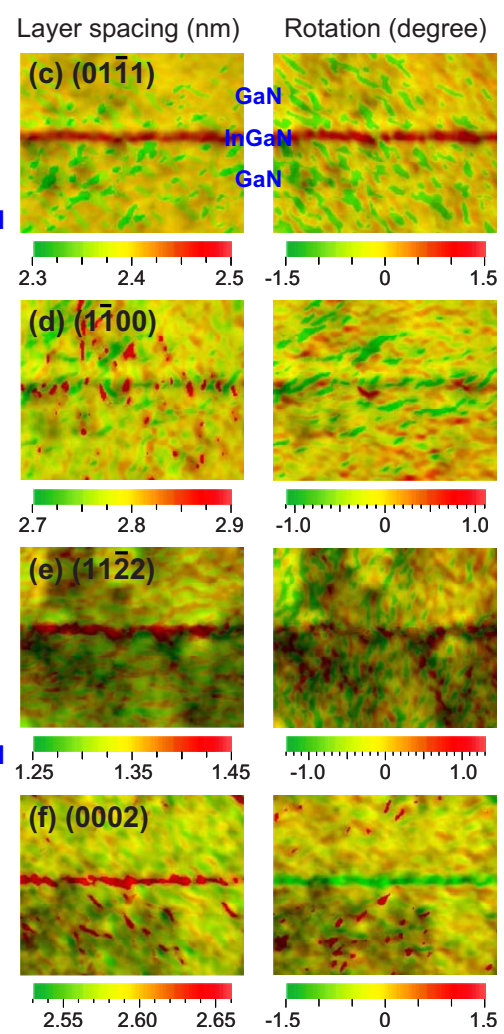
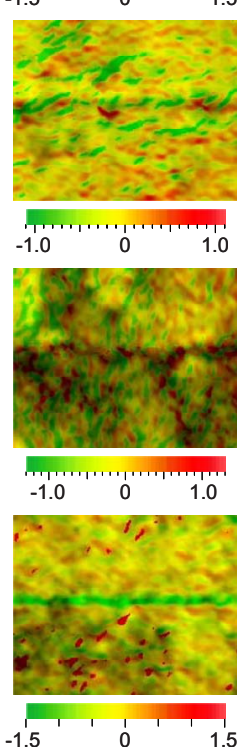

FIG. 4. (Color online) Cross sectional TEM high resolution images observed along (a) [ $\overline{1} \overline{1} 23]$ and $(b)[1 \overline{1} 00]$ directions. Markers represent $2 \mathrm{~nm}$. Inset in (a) is an example image after FFT, which is used to extract the layer spacing and rotation angle. (c)-(f) are those for the $(01 \overline{1} 1),(1 \overline{1} 00),(11 \overline{2} 2)$, and (0002) planes, respectively. Mappings (c), (d), and (e) are obtained by analyzing the corresponding spots designated in the FFT pattern, while the mapping (f) is obtained through FFT of (b). spacing and is rotated counterclockwise with respect to that in $\mathrm{GaN}$, both of which were clearly seen for the (01)1) plane in Fig. 4(c). Furthermore, the followings are confirmed.

- Figures 3(b) and 4(d): the (1100) planes in InGaN and $\mathrm{GaN}$ have the same spacing and are parallel because these are perpendicular to the interface.

- Figures 3(b), 3(c), and 4(e): the (11 22$)$ plane in InGaN has a larger layer spacing but is parallel to that in GaN.

- Figures 3(c) and 4(f): the (0001) plane in InGaN has a larger layer spacing and is rotated clockwise with respect to that in $\mathrm{GaN}$.

Thus, TEM supports the model developed from XRD.

\section{C. (1101) MQW}

Furthermore, to generalize the model, XRD-RSM was performed for another semipolar QW, that is, (1101) InGaN/

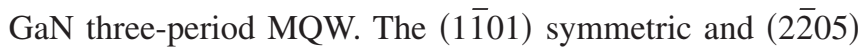
asymmetric diffractions were examined and we confirmed that the diffraction spots from GaN and InGaN were aligned along the surface normal in the reciprocal space, the same as those in Fig. 2.

\section{STRAIN TENSOR ELEMENTS FOR ARBITRARY CRYSTALLINE ORIENTATION}

The present model for a pseudomorphic interface relies on a very simple but persuasive criterion, that is, continuity of lattice planes across an interface. This idea has widely been accepted for well-defined planes such as $\{0001\}$ and nonpolar planes but has not explicitly been applied to semipolar planes. Herein, we present strain tensor elements that satisfies the criterion.

Unit vectors are denoted as $\hat{\bullet}$ and then the hexagonal primitive translation vectors under strain become ${ }^{3}$

$$
\left(\begin{array}{c}
\boldsymbol{\alpha}_{i} \\
\boldsymbol{\beta}_{i} \\
\boldsymbol{\gamma}_{i}
\end{array}\right)=\left(\begin{array}{ccc}
a_{i} & 0 & 0 \\
\frac{a_{i}}{2} & \frac{\sqrt{3} a_{i}}{2} & 0 \\
0 & 0 & c_{i}
\end{array}\right) \epsilon\left(\begin{array}{c}
\hat{\boldsymbol{x}} \\
\hat{\boldsymbol{y}} \\
\hat{\boldsymbol{z}}
\end{array}\right)
$$

where $a$ and $c$ are the wurtzite lattice parameters, $i$ represents the epilayer (e) or substrate (s), and

$$
\boldsymbol{\epsilon}=\left(\begin{array}{ccc}
1+\epsilon_{x x} & \epsilon_{x y} & \epsilon_{x z} \\
\epsilon_{y x} & 1+\epsilon_{y y} & \epsilon_{y z} \\
\epsilon_{z x} & \epsilon_{z y} & 1+\epsilon_{z z}
\end{array}\right) .
$$

$\epsilon_{i j}$ represents strain tensor elements. The condition for pseudomorphic interfaces determined by the XRD-RSM and TEM is that the projection of the reciprocal vectors on the interface must agree between the epilayer and substrate. Decomposing the projection into the in-plane $q_{x^{\prime}}$ and $q_{y^{\prime}}$ axes, this condition can be formulated using the inner products of the reciprocal vectors and the unit vectors for $q_{x^{\prime}}$ and $q_{y^{\prime}}$ axes $\left(\hat{\boldsymbol{q}}_{x^{\prime}}\right.$ and $\hat{\boldsymbol{q}}_{y^{\prime}}$, respectively) as follows:

$$
\begin{aligned}
& \boldsymbol{A}_{e} \cdot \hat{\boldsymbol{q}}_{x^{\prime}}=\boldsymbol{A}_{s} \cdot \hat{\boldsymbol{q}}_{x^{\prime}}, \\
& \boldsymbol{A}_{e} \cdot \hat{\boldsymbol{q}}_{y^{\prime}}=\boldsymbol{A}_{s} \cdot \hat{\boldsymbol{q}}_{y^{\prime}} .
\end{aligned}
$$

$\boldsymbol{A}_{i}$ is the reciprocal vector given by $\boldsymbol{\beta}_{i} \times \boldsymbol{\gamma}_{i} / V$, where $V$ $=\boldsymbol{\alpha}_{i} \cdot\left(\boldsymbol{\beta}_{i} \times \boldsymbol{\gamma}_{i}\right)$. Analogous formulae represent for $\boldsymbol{B}_{i}=\boldsymbol{\gamma}_{i}$ $\times \boldsymbol{\alpha}_{i} / V$ and $\boldsymbol{\Gamma}_{i}=\boldsymbol{\alpha}_{i} \times \boldsymbol{\beta}_{i} / V$. Noting that $\hat{\boldsymbol{q}}_{x^{\prime}}=\hat{\boldsymbol{x}}^{\prime}, \hat{\boldsymbol{q}}_{y^{\prime}}=\hat{\boldsymbol{y}}^{\prime}, \hat{\boldsymbol{q}}_{z^{\prime}}$ 
$=\hat{z}^{\prime}$, and the $\left(x^{\prime}, y^{\prime}, z^{\prime}\right)$ coordinate is related to the $(x, y, z)$ coordinate by the rotation matrix, then the following strain tensor elements are obtained:

$$
\begin{aligned}
& \epsilon_{x x}=\epsilon_{x x}^{(0)}+R \frac{\sin \theta}{\cos \theta} \epsilon_{x z}, \\
& \epsilon_{y y}=\epsilon_{x x}^{(0)},
\end{aligned}
$$

$$
\begin{aligned}
& \epsilon_{z z}=\epsilon_{z z}^{(0)}+R^{-1} \frac{\cos \theta}{\sin \theta} \epsilon_{x z}, \\
& \epsilon_{x y}=\epsilon_{y z}=0,
\end{aligned}
$$

where $\epsilon_{x x}^{(0)}=\left(a_{\mathrm{s}}-a_{\mathrm{e}}\right) / a_{\mathrm{e}}, \quad \epsilon_{z z}^{(0)}=\left(c_{\mathrm{s}}-c_{\mathrm{e}}\right) / c_{\mathrm{e}}, \quad$ and $\quad R=\left(\epsilon_{x x}^{(0)}\right.$ $+1) /\left(\epsilon_{z z}^{(0)}+1\right)$.

The remaining unknown strain tensor element of $\epsilon_{x z}$ can be extracted assuming traction-free surfaces; that is, the stress tensor element of $\sigma_{z^{\prime} z^{\prime}}$ is zero ${ }^{4-6,13}$

$$
\epsilon_{x z}=-\frac{\left\{\left(C_{11}+C_{12}+C_{13} \epsilon_{z z}^{(0)} / \epsilon_{x x}^{(0)}\right) \sin ^{2} \theta+\left(2 C_{13}+C_{33} \epsilon_{z z}^{(0)} / \epsilon_{x x}^{(0)}\right) \cos ^{2} \theta\right\} \epsilon_{x x}^{(0)} \sin \theta \cos \theta}{C_{11} R \sin ^{4} \theta+\left\{\left(R+R^{-1}\right) C_{13}+4 C_{44}\right\} \sin ^{2} \theta \cos ^{2} \theta+C_{33} R^{-1} \cos ^{4} \theta},
$$

where $C_{i j}$ represents elastic stiffness tensor elements. In the above calculation, the rotation axis was $y \|[1 \overline{1} 00]$. It was confirmed that even when the rotation axis is $x \|[\overline{1} 120]$, the result is essentially identical. [ $x$ and $y$ in Eqs. (4) and (5) are exchanged.] Therefore, the current model is applicable to arbitrary rotation axes.

To assess strain states in grown films, it is convenient to determine the strain tensor elements with respect to the $\left(x^{\prime}, y^{\prime}, z^{\prime}\right)$ coordinate. Rotating the strain tensor by $\theta$, the following expressions are obtained:

$$
\begin{aligned}
& \epsilon_{x^{\prime} x^{\prime}}=\epsilon_{x x} \cos ^{2} \theta+\epsilon_{z z} \sin ^{2} \theta-\epsilon_{x z} \sin 2 \theta \\
& \epsilon_{y^{\prime} y^{\prime}}=\epsilon_{y y}, \\
& \epsilon_{z^{\prime} z^{\prime}}=\epsilon_{x x} \sin ^{2} \theta+\epsilon_{z z} \cos ^{2} \theta+\epsilon_{x z} \sin 2 \theta \\
& \epsilon_{x^{\prime} z^{\prime}}=\left\{\left(\epsilon_{x x}-\epsilon_{z z}\right) \sin 2 \theta\right\} / 2+\epsilon_{x z} \cos 2 \theta .
\end{aligned}
$$

For the (0001) plane, a famous equation, $\epsilon_{z^{\prime} z^{\prime}}=\epsilon_{z z}=$ $-2 C_{13} / C_{33} \epsilon_{x x}^{(0)}$ was confirmed. Using $\epsilon_{z^{\prime} z^{\prime}}$, the layer spacing $d$ for a growth plane can be expressed as $d=\left(1+\epsilon_{z^{\prime} z^{\prime}}\right) d^{(0)}$, where $d^{(0)}$ is the layer spacing without strain.

\section{DISCUSSION}

There are differences between our model and the earlier models. ${ }^{3,5}$ In Ref. 3, the shear component of $\epsilon_{x z}$ was determined by minimizing the strain energy. This procedure looks inconsistent to ours, but we found that their proposed strain tensor elements simultaneously satisfy the traction-free surface condition. Therefore, the remaining difference is our criteria; Ref. 3 assumed that the projections of the primitive translation vectors of the epilayer and the substrate on the interface were equal. This assumption appeared unreasonable from our experimental results. Nevertheless, the quantitative difference between the two models is negligible because replacing $R$ in our model with unity produces their model and $R$ for pseudomorphic InGaN on unstrained $\mathrm{GaN}$ is between
0.992 (for $\mathrm{InN}$ ) and 1 (for GaN). Furthermore, for planes without a shear component such as $\{0001\}$ and nonpolar planes, the two models are identical.

On the other hand, the strain tensor elements for $\mathrm{In}_{0.2} \mathrm{Ga}_{0.8} \mathrm{~N} / \mathrm{GaN}$ were calculated as a function of crystal angle $(\theta)$ to directly compare with Fig. 5(a) in Ref. 5. Figure 5 shows the result. ${ }^{15}$ All the physical parameters for $\mathrm{GaN}$ and InN were the same as those in Ref. 5, and those for InGaN were extracted by linear interpolation. Although Fig. 5 shows the result for $\mathrm{In}_{0.2} \mathrm{Ga}_{0.8} \mathrm{~N} / \mathrm{GaN}$ as an example, it was confirmed that changing the In composition up to $100 \%$ just alter the magnitude of strain components without affecting the essential characteristics. The most noticeable difference between the models is in $\epsilon_{x^{\prime} z^{\prime}}$. The reason for the observed difference in the shear strain components is presently unclear, but it might be related to the definition of their $\epsilon_{y^{\prime} y^{\prime}}$ (that is, our $\epsilon_{x^{\prime} x^{\prime}}$ ) under the presence of misalignment of the crystal orientations between the strained and unstrained materials, as demonstrated in Figs. 3 and 4.

A question may arise as to if the difference in the strain tensor elements due to the two models is experimentally detectable in XRD-RSM and/or TEM. To answer this question, reciprocal vectors were calculated from the strain tensor elements proposed in this study and in Ref. 5. It was confirmed that the two models cause no difference in the components

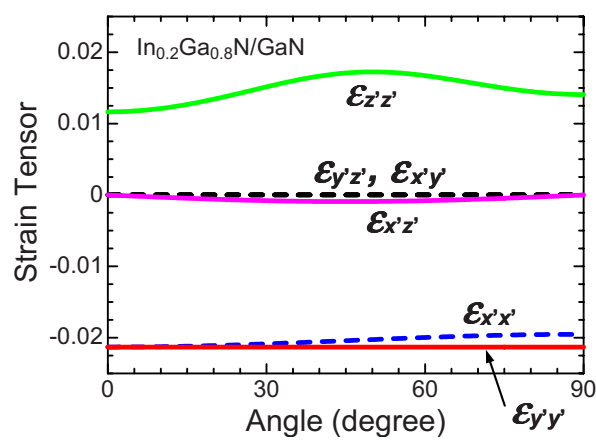

FIG. 5. (Color online) Strain tensor elements for $\operatorname{In}_{0.2} \mathrm{Ga}_{0.8} \mathrm{~N} / \mathrm{GaN}$ with respect to the growth plane. Positive and negative tensor elements indicate tensile and compressive strain, respectively. 


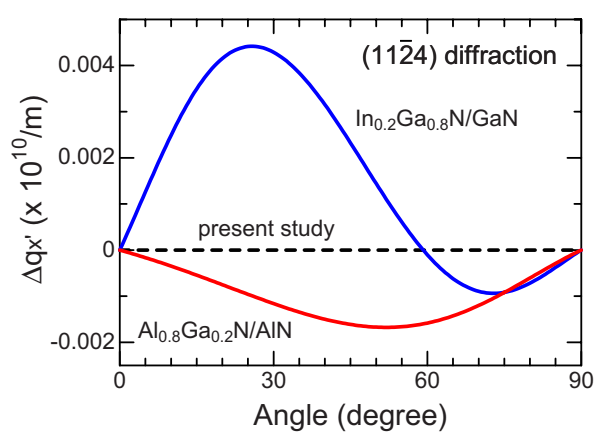

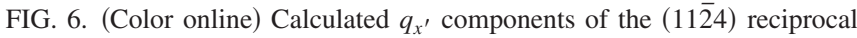
vectors for $\operatorname{In}_{0.2} \mathrm{Ga}_{0.8} \mathrm{~N} / \mathrm{GaN}$ and $\mathrm{Al}_{0.8} \mathrm{Ga}_{0.2} \mathrm{~N} / \mathrm{AlN}$ pseudomorphic heterostructures as a function of crystal angle. $\Delta q_{x^{\prime}}$ represents shift in $q_{x^{\prime}}$ from unstrained GaN or AlN. The (blue and red) thick solid lines for InGaN and $\mathrm{AlGaN}$, respectively, are based on the model proposed in Ref. 5, whereas the (black) broken line, which is for both $\mathrm{InGaN}$ and $\mathrm{AlGaN}$, is based on our model.

along the $q_{y^{\prime}}$ axis of reciprocal vectors. This is because $q_{y^{\prime}}$ is the rotation axis, along which the strain tensor elements, that is, our $\epsilon_{y^{\prime} y^{\prime}}$ and their $\epsilon_{x^{\prime} x^{\prime}}$ are identical. On the other hand, the $q_{x^{\prime}}$ and $q_{z^{\prime}}$ components depend on the models. Figure 6 shows the results of example calculations for $\operatorname{In}_{0.2} \mathrm{Ga}_{0.8} \mathrm{~N}$ pseudomorphically grown on unstrained $\mathrm{GaN}$ as well as $\mathrm{Al}_{0.8} \mathrm{Ga}_{0.2} \mathrm{~N}$ pseudomorphically grown on unstrained $\mathrm{AlN}$, an important strucuture for deep ultraviolet emitters. Here, the $q_{x^{\prime}}$ components of the $(11 \overline{2} 4)$ reciprocal vectors were calculated, and differences between $q_{x^{\prime}}$ of the strained and unstrained layers $\left(\Delta q_{x^{\prime}}\right)$ are plotted as a function of crystal angle. If $\Delta q_{x^{\prime}}$ is not zero, the diffraction spots are not aligned vertically in the reciprocal space, and consequently, the planes are not continuous at the interface (see Figs. 2 and 3). Because one of our criteria to formulate the strain states is the vertical alignment of the diffraction spots in the reciprocal space, our model satisfies $\Delta q_{x^{\prime}}=0$ in principle for any of pseudomorphic heterostructures including $\mathrm{In}_{0.2} \mathrm{Ga}_{0.8} \mathrm{~N} / \mathrm{GaN}$ and $\mathrm{Al}_{0.8} \mathrm{Ga}_{0.2} \mathrm{~N} / \mathrm{AlN}$, as recognized by the (black) broken line in Fig. 6. In contrast, the model in Ref. 5 provides nonzero values, as shown by the (blue and red) thick solid curves in Fig. 6. It is noteworthy that $\Delta q_{x^{\prime}}>\sim 0.002 \times 10^{10} / \mathrm{m}$ should clearly be recognized in XRD-RSMs as a displacement from the vertical alignment. On the other hand, the largest $\Delta q_{x^{\prime}}$ for $\operatorname{In}_{0.2} \mathrm{Ga}_{0.8} \mathrm{~N} / \mathrm{GaN}$ corresponds to a shift in approximately $0.1 \mathrm{~nm}$ in TEM high resolution lattice images. Therefore, very careful TEM analyses are required to see the difference due to the models, and XRD-RSM and TEM measurements should be complementary; XRD-RSM assesses a quite limited number of reciprocal vectors with very high accuracy, while TEM provides information on many reciprocal vectors at the same time, but its resolution is not so high as XRD.

The difference due to the two models appears also in the electronic band structure. For example, the topmost valence band (A band) energies were calculated, using the $6 \times 6$ valence band Hamiltonian for strained wurtzite semiconductors. ${ }^{3,16}$ It was found that the two models cause energy differences of more than $15 \mathrm{meV}$ for a semipolar $\mathrm{In}_{0.2} \mathrm{Ga}_{0.8} \mathrm{~N} / \mathrm{GaN}$ pseudomorphic heterostructure tilted $25^{\circ}$ with respect to the (0001) plane, and furthermore, $30 \mathrm{meV}$ for a semipolar $\mathrm{Al}_{0.8} \mathrm{Ga}_{0.2} \mathrm{~N} / \mathrm{AlN}$ pseudomorphic heterostructure tilted $50^{\circ}$ with respect to the (0001) plane. (Note that those angles correspond to the angles that cause the largest $\Delta q_{x^{\prime}}$ in Fig. 6.) Because a valence band discontinuity at $\mathrm{In}_{0.2} \mathrm{Ga}_{0.8} \mathrm{~N} / \mathrm{GaN}$ is $\sim 135 \mathrm{meV}$ and that at $\mathrm{Al}_{0.8} \mathrm{Ga}_{0.2} \mathrm{~N} / \mathrm{AlN}$ is $\sim 170 \mathrm{meV}$, the variations due to the models are not negligible. Precisely determining strain states is thus important to understanding the optical properties of semipolar, pseudomorphic nitride semiconductor QWs. It should be emphasized that our model is derived from the experimental results and, therefore, seems more reliable.

\section{SUMMARY}

Strain in wurtzite III-nitride semiconductor QWs was investigated using semipolar InGaN/GaN QWs. Experimentally, the XRD-RSM and TEM analysis demonstrated that layers are not interrupted at pseudomorphic interfaces. Based on this simple conclusion, the strain tensor elements were formulated for arbitrary crystalline orientation. It is noteworthy that the current procedure is quite versatile and can easily be expanded to any crystal structures by replacing our Eq. (1) with appropriate primitive translation vectors; for zincblende structures, for example, Eq. (3) in Ref. 14 can be used.

\section{ACKNOWLEDGMENTS}

This work is partially supported by a Grant-in-Aid for Scientific Research (A) (19206036) and the Global COE Program by the Ministry of Education, Culture, Sports, Science, and Technology of Japan.

${ }^{1}$ G. L. Bir and G. E. Pikus, Symmetry and Strain-Induced Effects in Semiconductors (Wiley, New York, 1974).

${ }^{2}$ J. F. Nye, Physical Properties of Crystals (Oxford Science, New York, 1957).

${ }^{3}$ S.-H. Park and S.-L. Chuang, Phys. Rev. B 59, 4725 (1999).

${ }^{4}$ T. Takeuchi, H. Amano, and I. Akasaki, Jpn. J. Appl. Phys., Part 1 39, 413 (2000).

${ }^{5}$ A. E. Romanov, T. J. Baker, S. Nakamura, and J. S. Speck, J. Appl. Phys. 100, 023522 (2006).

${ }^{6}$ B. Gil, P. Bigenwald, and O. Briot, Phys. Status Solidi (RRL) 1, 268 (2007).

${ }^{7}$ K. Nishizuka, M. Funato, Y. Kawakami, Sg. Fujita, Y. Narukawa, and T. Mukai, Appl. Phys. Lett. 85, 3122 (2004).

${ }^{8}$ B. Neubert, P. Brückner, F. Habel, F. Scholz, T. Riemann, J. Christen, M. Beer, and J. Zweck, Appl. Phys. Lett. 87, 182111 (2005).

${ }^{9}$ M. Ueda, K. Kojima, M. Funato, Y. Kawakami, Y. Narukawa, and T. Mukai, Appl. Phys. Lett. 89, 211907 (2006).

${ }^{10}$ M. Funato, M. Ueda, Y. Kawakami, Y. Narukawa, T. Kosugi, M. Takahashi, and T. Mukai, Jpn. J. Appl. Phys., Part 2 45, L659 (2006).

${ }^{11}$ H. Sato, R. B. Chung, H. Hirasawa, N. Fellows, H. Masui, F. Wu, M. Saito, K. Fujito, J. S. Speck, S. P. DenBaars, and S. Nakamura, Appl. Phys. Lett. 92, 221110 (2008).

${ }^{12}$ M. Ueda, M. Funato, K. Kojima, Y. Kawakami, Y. Narukawa, and T. Mukai, Phys. Rev. B 78, 233303 (2008).

${ }^{13}$ D. Sun and E. Towe, Jpn. J. Appl. Phys., Part 1 33, 702 (1994).

${ }^{14}$ D. L. Smith and C. Mailhiot, J. Appl. Phys. 63, 2717 (1988).

${ }^{15}$ Note that $x^{\prime}\left(y^{\prime}\right)$ in Ref. 5 is $y^{\prime}\left(x^{\prime}\right)$ in this paper.

${ }^{16}$ M. Kumagai, S. L. Chuang, and H. Ando, Phys. Rev. B 57, 15303 (1998). 